\title{
Design and Implementation of Smart Gateway Based on Wireless Communication Technique
}

\author{
Zhongxiang Xiao ${ }^{1, a}$, Tengxue Rong ${ }^{1, b}$, Guojian Cheng ${ }^{2, c}$, Xinjian Qiang ${ }^{2, d^{*}}$ and \\ Ye Liü, e \\ ${ }^{1}$ School of Electronic Engineering, Xi'an Shiyou University, Xi'an Shaanxi, China \\ ${ }^{2}$ School of Computer Science, Xi'an Shiyou University, Xi'an Shaanxi, China

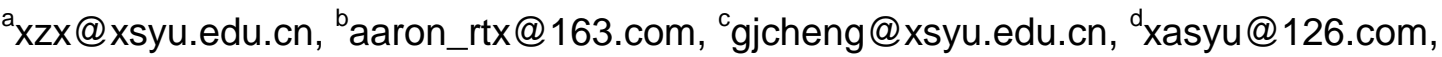 \\ yeliu@xsyu.edu.cn \\ *The corresponding author
}

Keywords: ZigBee; CC2530; Smart gateway; Arduino

\begin{abstract}
A large number of advanced technology dedicated to research and development smart home applications in the IOT environment, especially using of open-source Arduino and ZigBee wireless communication protocol to build a interoperability smart home system have major value. In the designing of this smart home, researching on the characteristics of ZigBee technology and network topology, making a special effort to analyze the ZigBee protocol specification.It mainly studied and designed the smart gateway based on CC2530 and Arduino, that realize the communication of each devices, and achieve the remote monitoring and wireless control in the home. In addition, the performance test and power analysis are completed, which provides a strong guarantee for the further development of smart home.
\end{abstract}

\section{Introduction}

Smart home is a platform of using residents' housing that connected household appliances by use of computer networks, communications technology and automation technology, which can provide a mo safer, comfortable, efficient and convenient living environment. As early as 1998, Bill Gates has built a smart home for himself. Smart home Connected with lines in the early, which limits its own development largely, and the price is expensive, not the general public consumer goods [1]. Wireless solutions has brought the dawn for home automation, cutting off the cumbersome lines, that is conducive to further development and application. Wireless communication technology is the way of communication that mainly uses electromagnetic waves rather than the cable. At present, there is much mainstream wireless communication technology in study that mainly includes: bluetooth, WiFi, USB, NFC, and ZigBee.

ZigBee has a strong advantage to build network, it can reach to 65000 nodes in theoretically. Global industrial, scientific and medical frequency band is the most common $2.4 \mathrm{GHz}$. ZigBee is the application of the frequency band protocol. In the smart home, ZigBee can provide a complete routing function, so it compensates for the weak penetration. Arduino use electronic building block type to development, it very convenient for the production of a variety of prototype sensor, to solve the problem that a variety of sensors and terminal equipment access standards are not consistent. Android also provides a good man-machine interface. ZigBee, Arduino and Android combination for the development of smart home applications will create infinite possibilities, it play a great role in promoting the development of smart home and IOT. This article will combine these techniques to design a smart home smart gateway. 


\section{Technical Overview}

Arduino. Arduino is derived from the Italian that is an open-source electronics prototyping platform of a open source electronic hardware project, which comprises a simple I/O functions of circuit board and an open source software development environment, not only can run independently, but also with the nature of interaction. Users can control the Arduino board through relatively simple programming language, combined with all kinds of sensors and the function modules to achieve more functions of perception, recognition, processing and other [2]. Arduino with the characteristic of open-source and easy to use provides a good platform for the prototype design of intelligent products. With the increase of users and peripheral products, Arduino has formed an open source ecosystem, the rich software and hardware resources can meet the needs of the vast most intelligent product prototype design. However, Arduino are not design and development for intelligent product prototype specifically, the user is likely to feel confused and overwhelmed when face a large number of available resources. Therefore, studying the prototype design of intelligent products were in-depth, and blending in the advantage of Arduino and ecosystem into them, that has important significance for improving the role of smart product prototypes in designing the intelligent products.

Wireless Sensor Technology of ZigBee. ZigBee wireless sensor technology is a two-way wireless communication technology that is short distance, low power, low complexity, low cost and low rate, it mainly used in a variety of electronic devices is low data transmission between each other which is near distance, low power and transmission rate, as well as applied to the data transmission of typical intermittent data, cyclical data and the data of reaction time is low. The world has never been cracked precedent for the ZigBee security. ZigBee uses AES advanced encryption system, the degree of secrecy is equivalent to 12 times the bank card encryption technology [3]; Secondly, the ZigBee network uses a cellular structure, each device and gateway communication is multi direction, which have a high network stability; The nodes of network capacity is 65000 in theory that enough to meet the needs of home network coverage, even intelligent residential, intelligent buildings and other also can be fully covered; Finally, ZigBee has the capabilities of two-way communication, that is not only can send command to the device, not only can send commands to the device, but also the device is able to feedback the perform state back, which is critical to the user experience, especially security equipment. In addition, ZigBee uses a very low power design that can be supply power by fully battery, battery can be used for more than 10 years in theory, also can achieve the purpose of energy conservation and environmental protection [4].

\section{The Hardware Design of Smart Gateway}

The Hardware Structure of Smart Gateway. Intelligent gateway plays an important role in intelligent home system, and it acts as a good bridge between communications networks outside the family and control networks within the family [5]. The whole hardware structure design of the gateway platform as shown in the Fig. 1, which mainly includes the following sections: the control kernel module of Arduino, the man-machine interaction module, storage system, the power-supply module, the network module, the communication module and the extension interface.

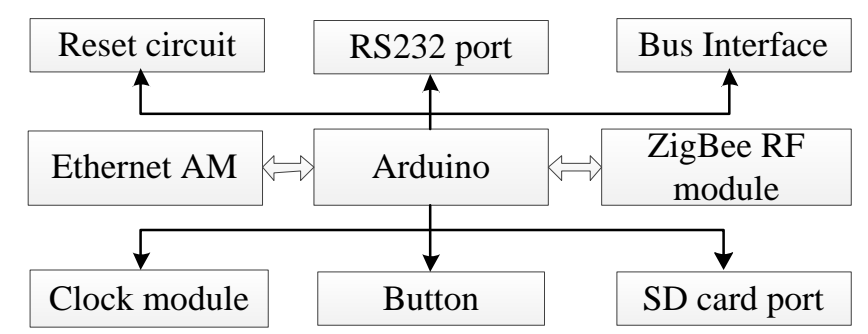

Figure 1. The frame diagram of the Smart Gateway 
From the hardware structure charts, we can find that Arduino module with W5100 is the core of the main module. Take it as the core to open to expand the other hardware module that through serial port and USB serial bus to communicate, to achieve the purpose of cooperative work. The W5100 is a multifunctional single chip network interface chip, and it can let embedded devices complete network communication fast under the conditions of low system resources occupation [6]. Because of integrating the following three functions: the TCP/IP protocol stack, the MAC and PHY of Ethernet, the W5100 can make various single chip microcomputers access the network simply and reliably without the support of the operating system. The ZigBee module provides the interactive communication interface between the home gateway and the ZigBee network within the family, and the customers can access the ZigBee network within the family by the home gateway and monitor the household electronic products which have ZigBee node. The peripheral equipment and extension modules are mainly used to completing the address decode of some equipment and external communication task, as well as providing the reserved interface for system upgrade later.

The Design of Communication Circuit. ZigBee communication module is the basic unit in the family network that connected the home network and the Internet to achieve the data forwarding through the ZigBee communication module. The CC2530 8051 kernel is able to work together with ZigBee wireless circuit that in the $2.4 \mathrm{GHZ}$ band. CC2530 has high degree of integration, so it must be connected components is very small, usually just a crystal oscillator can be meet the wireless transceiver and network requirements [7]. The design of hardware circuit schematic for communication is shown in Fig. 2.

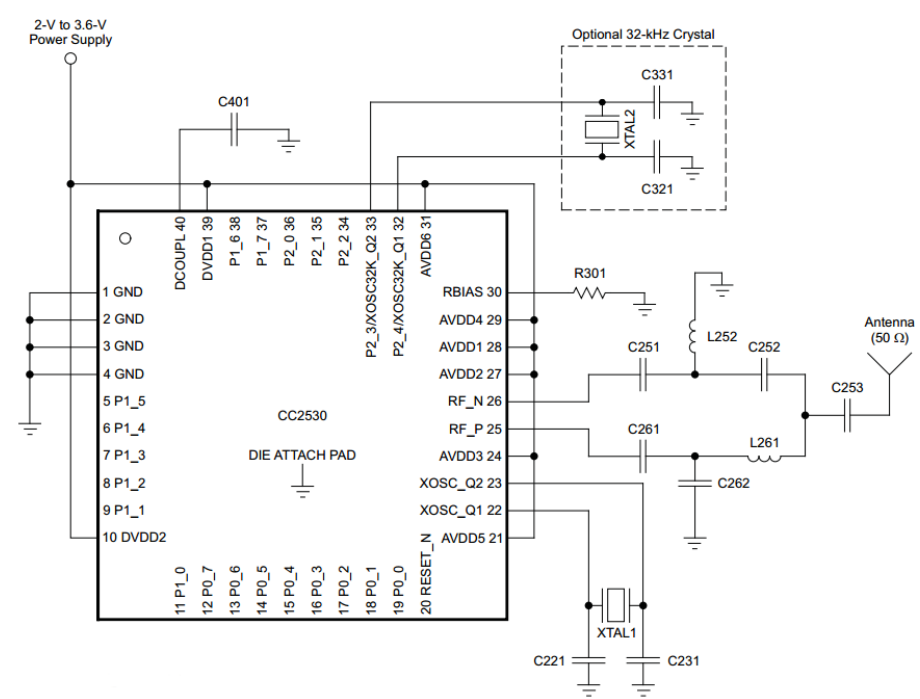

Figure 2. The Schematic diagram of CC2530 Communication

\section{The Software Design of Smart Gateway}

System Architecture of Smart Gateway. According to the system design requirements, the intelligent gateway is not only to run the TCP/IP protocol, but also to run the ZigBee protocol. The software system architecture of the intelligent gateway is shown in Fig. 3, the system software adopts the layered modular architecture, each layer of software can complete some functions independently, and provide services to other levels through the interface function [8]. Located at the bottom is the hardware driver, the driver includes the gateway ZigBee and Arduino all the hardware driver code. On the HAL layer is the hardware abstraction layer, the hardware abstraction layer is the Arduino core processing system, which is based on the TCP/IP protocol stack and the ZigBee protocol stack. The top layer is the application layer to realize the intelligent gateway architecture, to achieve human-computer interaction [9]. 


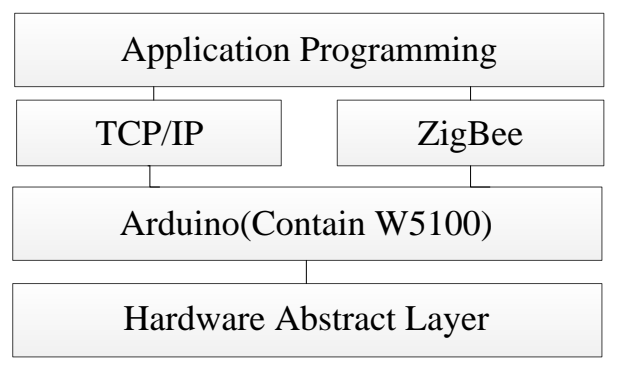

Figure 3. The network structure of gateway System

Application Design of Software. ZigBee protocol stack running on the OSAL operating system, OSAL is based on the task scheduling mechanism, it respond to an event number through the task of the event trigger. Protocol stack flow chart is shown in Fig. 4. When an event occurs, such as a button, which will call the response function of the button to generate a task, and then through the task to send the function of "osal_msg send", send a message processing cycle, and register the event, wait for processing [10]. In the application layer, when the event is detected by its processing function that will call the corresponding processing function.

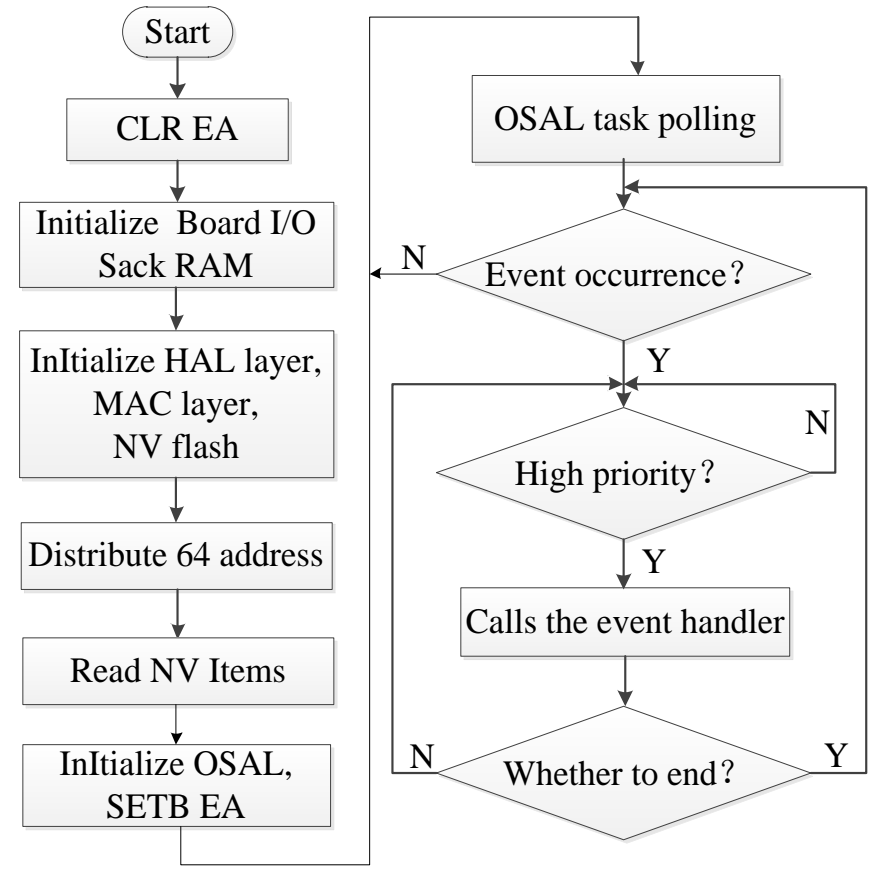

Figure 4. The flow chart of protocol stack

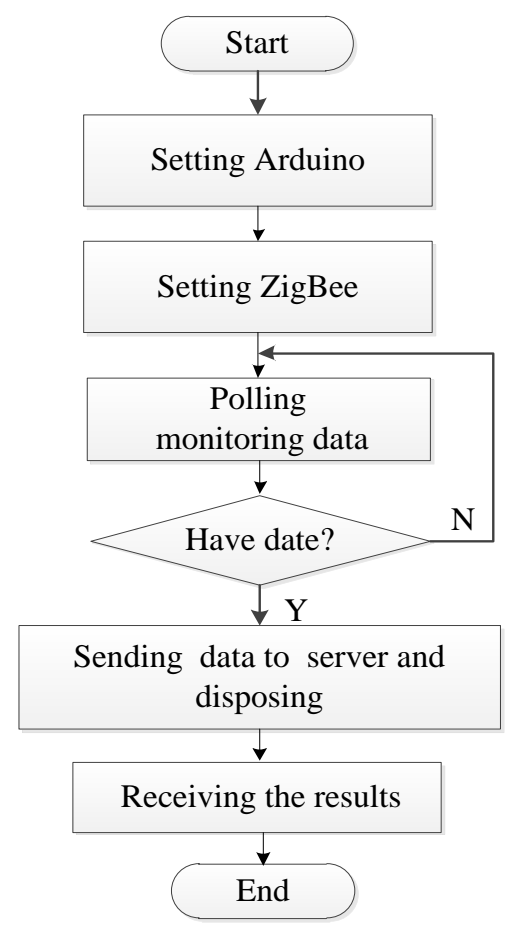

Figure 5. The program flow chart of Arduino

Arduino motherboard contains W5100 in the system that integrates LwIP which is the TCP/IP open source protocol stack, after installing a driver that would be transplanted to the Z-Stack LwIP, all the TCP/IP protocol of LwIP protocol stack in a same process. As shown in Fig. 5 that is program flow chart of Arduino motherboard. The function of ZigBee terminal equipment is mainly to receive the coordination of the control commands issued, such as switching the home power, at the same time we can know that the operation of home appliances through the coordinator. Terminal equipment is in a state of sleep at the most of the time, they will recover from the sleep when it need to deal with the task, and once again into the sleep state after the task processing.

\section{The Test of Smart Gateway}

Smart home system gateway will be started in the form of a coordinator or router to join the family of internal network, and connected internet through the TCP/IP. Users will be able to achieve a variety of 
applications through using the browser, that will achieve the intelligent monitoring and managing to the household appliances. Gateway testing is mainly divided into three steps, first of all is installing the drive of CP2102, one-to-one connected CP2102 and the Arduino board, the power is supplied by the USB, the next to download program for the Arduino board, after code loading, plugged in cable, then open the serial network to test weather is successful. Secondly, putting ZigBee module into ZigBee development board, building IAR environment, then connecting the downloader and install the drive connecting, compiling and downloading the ZigBee networking code. Next to connect a LED in the board of ZigBee development (P1.0). Finally, connecting ZigBee development and Arduino board, and test by using mobile phone control end. Opening the web site "m.znck007.com" by a mobile phone, creating a new switch and click it, if LED lights flashing, so gateway is normal, then you can create network to realize the intelligent controlling.

\section{Summary}

At present, there are a lot of scheme of intelligent home system that they are too complex and closed. This paper mainly designed and realized smart home gateway hardware and software based on ZigBee wireless sensor technology and open-source Arduino platform. Applied smart home gateway system to the home environment and testing the function and communication distance, we can analyzed the test results that will provide evidence for the application feasibility that wireless smart home gateway system used in the family. Due to a short period of time that only designed and realized the prototype system according to the existing techniques. Many aspects are lack of consideration, and next step will continue to study ZigBee protocol, to improve the smart home and wireless network interoperability by use more perfect and stable solutions.

\section{Acknowledgements}

The Key Project Supported by Industrial Science and Technology in Shaanxi Province under Grant No. 2015GY104. The Project Supported by Natural Science Basic Research Plan in Shaanxi Province of China under Grant No. 2014JQ5193. The Project Supported by Youth Science and Technology Innovation Fund of Xi'an Shiyou University under Grant No. 2014BS13.

\section{References}

[1] Z.B. Ji: Telkomnika - Indonesian Journal of Electrical Engineering (Wenzhou, China, March 14, 2013). Vol.11, p.4414.

[2] X.Q. Wang, Y.J. Ou and N.L. Huang: The Design and Implementation of ZigBee Wireless Sensor Network (Chemical Industry Press, China 2012).

[3] N. Yang: Design and study Smart product prototypes based on Arduino (MS., Jiangnan University, China 2014).

[4] Texas Instruments. Z-Stack sample application for SmartRF05 and CC2530 on http:// www .ti.com/lsds/ti/tools-software/wireless_sw.page.

[5] Z.H. Qian and Y.J. Wang: Journal of Electronics \& Information Technology, Vol.35 (2013) No.1, p.215-227.

[6] H.L. WU: Study Smart Home Gateway Based on Embedded System (MS., Zhejiang University, China 2006).

[7] E. Cano, I. Garcia: IEEE Electronics, Robotics and Automotive Mechanics Conference (Cuernavaca, Morelos, Mexico, November 15-18. 2011).p.366.

[8] D.Y. Jin. Application Research of ZigBee Technology in Smart Home Control System (MS., Taiyuan University of Technology, China 2008). 
[9] Z-Stack ZigBee Protocol Stack on http://www.com/tool/z-stack.

[10] J.Q. Li, S.Q.He and Z. Ming: Journal of Shenzhen University Science and Engineering, Vol.31 (2014) No.6, p.630. 